\title{
Nonlinear Civil Structures Identification Using a Polynomial Artificial Neural Network
}

\author{
Francisco J. Rivero-Angeles ${ }^{1, \star}$, Eduardo Gomez-Ramirez ${ }^{2}$, and Ruben Garrido ${ }^{1}$ \\ 1 Centro de Investigacion y de Estudios Avanzados del IPN, \\ CINVESTAV - Departamento de Control Automatico, \\ Av. Instituto Politecnico Nacional \#2508, Col. Zacatenco, \\ A.P. 14-740, Mexico, D.F. 07360, Mexico \\ 2 Universidad La Salle - Laboratorio de Investigacion y Desarrollo, \\ de Tecnologia Avanzada. Benjamin Franklin \#47, Col. Condesa, \\ Mexico, D.F. 06140, Mexico \\ frivero@candeingenieros.com
}

\begin{abstract}
Civil structures could undergo hysteresis cycles due to cracking or yielding when subjected to severe earthquake motions or even high wind. System identification techniques have been used in the past years to assess civil structures under lateral loads. The present research makes use of a polynomial artificial neural network to identify and predict, online, the behavior of such nonlinear structures. Simulations are carried out using the Loma Prieta and the Mexico City seismic records on two hysteretic models. Afterwards, two real seismic records acquired on a 24-story concrete building in Mexico City are used to test the proposed algorithm. Encouraging results are obtained: fast identification of the weights and fair prediction of the output acceleration.
\end{abstract}

\section{Introduction}

Health monitoring of structures has been a focus of interest for researchers in structural and control engineering for the past two decades. Civil structures, such as buildings and bridges, are instrumented to acquire output acceleration, velocity and displacement data due to lateral loads, which could be severe wind or strong earthquake motions. The data is later analyzed to assess the lateral resistant capacity of the structure and to check output maximums against those allowed by construction codes. In some instances, wind or earthquake forces may induce lateral loads to civil structures such that energy may dissipate through hysteretic phenomena, a nonlinear time-variante behavior which reduces their resistant capacity [5]. Many buildings have been instrumented around the world in order to monitor their structural health. The identification of such nonlinear systems is therefore an important task for engineers who work in areas affected by these natural hazards, and thus, the subject of the present paper.

Forecasting time series has been solved with a broad range of algorithms such as ARMAX [1], NARMAX [2], Fuzzy Logic [14], Neural Networks 3], etc. Some

\footnotetext{
^ Corresponding author.
} 
researchers have succesfully identified nonlinear structures with a wide variety of proposed algorithms 4]. Some examples include: ERA-OKID, Subspace and Least Squares algorithms to estimate linear parameters of structures [9]. An Orthogonal NARMAX model is proposed in [6]. Sequential regression analysis, Gauss Newton optimization and Least Squares with extended Kalman filter is reviewed in [8]. Least Squares methods have also been used by [10], 13], and [16].

Although artificial neural networks have not been widely used in civil and structural engineering, some researchers have succesfully applied them ([1], [12, and [7]). Nonetheless, the models and architectures of those networks seem quite complex and computer time consuming.

The present research proposes the use of a polynomial artificial neural network [3] to identify a nonlinear structural system with a fairly small amount of samples for on-line training. One important issue to consider is the use of on-line algorithms for closed-loop control applications or simulation and fault detection analysis, that is the reason an on-line algorithm is proposed.

In the present research, the Loma Prieta (California, USA, 1989) and SCT (Mexico City, Mexico, 1985) seismic records are used to test the proposed algorithm on a hysteretic simulated shear building structure. A Bouc-Wen model [15] is used to simulate a hysteretic nonlinear single degree of freedom structure (SDOF). Simulation results show that the proposed network is able to identify the nonlinear system and predict with good accuracy the acceleration output with a fairly simple model. Later on, one actual seismic record, acquired on a real 24-story concrete structure in Mexico City in 2002, is used to identify the behavior of the building. The identified model is then used to predict the acceleration motion of the same real building, subjected to another actual record acquired ten months later, in 2003, and the results show that this simple model predicts with very good accuracy the behavior of the system.

The proposed network model has two interesting features: (1) the driving external forces are considered unknown and not needed, which for the case of wind loading this model is applicable; and (2) this model does not need physical structural parameters, which in turn is a nice advantage when an instrumentation is set up in an unknown structural system. A long term aim of the present research is to develop a technique that could be used in conjunction with fault detection analysis, structural health monitoring, and structural control.

\section{Polynomial Artificial Neural Network}

The model of a polynomial artificial neural network (PANN) is shown in (1).

$$
\begin{aligned}
\hat{y}_{k}= & {\left[\phi \left(x_{1, k}, x_{2, k}, \ldots, x_{n_{i}, k}, x_{1, k-1}, x_{2, k-1}, \ldots,\right.\right.} \\
& \left.\left.x_{n_{i}, k-n_{1}}, \ldots, y_{k-1}, y_{k-2}, \ldots, y_{k-n_{2}}\right)\right]_{\phi_{\min }}^{\phi_{\max }} ;
\end{aligned}
$$

where $\hat{y}_{k} \in \Re$ is the estimated time series, $\phi(x, y) \in \Re$ is a nonlinear function, $x_{i} \in X$ are the inputs for $i=1, \ldots, n_{i}$; and $n_{i}$ is the number of inputs. $y_{k-j} \in Y$ are the previous values of the output, for $j=1, \ldots, n_{2} ; n_{1}$ is the number of 
delays of the input, $n_{2}$ is the number of delays on the output, $X$ and $Y$ are compact subsets of $\Re$. Simplifying the notation, it results into (2).

$$
\begin{array}{r}
z=\left(x_{1, k}, x_{2, k}, \ldots, x_{n_{1}, k}, \ldots, y_{k-1}, y_{k-2}, \ldots, y_{k-n_{2}}\right) \\
z=\left(z_{1}, z_{2}, z_{3}, \ldots, z_{n_{v}}\right) ;
\end{array}
$$

where $n_{v}$ is the total number of elements in description $z$, and $n_{v}=n_{i}+n_{1} n_{i}+n_{2}$. The nonlinear function $\phi(z) \in \Phi_{p}$ belongs to a family $\Phi_{p}$ of polynomials that can be represented as (3)

$$
\begin{aligned}
\Phi_{p}\left(z_{1}, z_{2}, \ldots, z_{n_{v}}\right)=(\phi(z): & \phi(z)=a_{0}\left(z_{1}, z_{2}, \ldots, z_{n_{v}}\right)+a_{1}\left(z_{1}, z_{2}, \ldots, z_{n_{v}}\right) \\
& \left.+a_{2}\left(z_{1}, z_{2}, \ldots, z_{n_{v}}\right)+\ldots+a_{p}\left(z_{1}, z_{2}, \ldots, z_{n_{v}}\right)\right)
\end{aligned}
$$

The subindex $p$ is the maximum power of the polynomials expression and $a_{i}\left(z_{1}, z_{2}, \ldots, z_{n_{v}}\right)$ are homogeneous polynomials of total degree $i$, for $i=0, \ldots, p$. Every homogeneous polynomial could be written as shown in (4)

$$
\begin{array}{r}
a_{0}\left(z_{1}, z_{2}, \ldots, z_{n_{v}}\right)=w_{0} \\
a_{1}\left(z_{1}, z_{2}, \ldots, z_{n_{v}}\right)=w_{1,1} z_{1}+w_{1,2} z_{2}+\ldots+w_{1, n_{v}} z_{n_{v}} \\
a_{2}\left(z_{1}, z_{2}, \ldots, z_{n_{v}}\right)=w_{2,1} z_{1}^{2}+w_{2,2} z_{1} z_{2}+\ldots+w_{2, N_{2}} z_{n_{v}}^{2} \\
\vdots \\
a_{p}\left(z_{1}, z_{2}, \ldots, z_{n_{v}}\right)=w_{p, 1} z_{1}^{p}+w_{p, 2} z_{1}^{p-1} z_{2}+\ldots+w_{p, N_{p}} z_{n_{v}}^{p} ;
\end{array}
$$

where $w_{i, j}$ is the associated weight of the network. The term $w_{0}$ corresponds to the input bias of the network. The homogeneous polynomial $a_{1}(z)$ is equivalent to weight the inputs. The polynomials $a_{2}(z)$ to $a_{p}(z)$ represent the modulation between the inputs and the power of each polynomial. $N_{i}$ is the number of terms of every polynomial with:

$$
\begin{array}{r}
N_{0}=1 ; N_{1}=n_{v} ; N_{2}=\sum_{i=1}^{n_{v}} i ; N_{3}=\sum_{s_{1}=0}^{n_{v}-1} \sum_{i=1}^{n_{v}-s_{1}} i ; \ldots \\
\ldots ; N_{p}=\underbrace{\sum_{s_{p-2}=0}^{n_{v}-1} \cdots \sum_{s_{2}=0}^{n_{v}-s_{3}} \sum_{s_{1}=0}^{n_{v}-s_{2}} \sum_{i=1}^{n_{v}-s_{1}}}_{p-1} i .
\end{array}
$$

The dimension of $N_{\Phi}$ of each family $\Phi_{p}$ could be computed by $N_{\Phi}=\sum_{i=0}^{p} N_{i}$. The activation function is given by (6)

$$
[\phi(z)]_{\phi_{\min }}^{\phi_{\max }}=\left\{\begin{array}{cc}
\phi_{\max } & \phi(z) \geq \phi_{\max } \\
\phi(z) & \phi_{\min }<\phi(z)<\phi_{\max } \\
\phi_{\min } & \phi(z) \leq \phi_{\min }
\end{array}\right.
$$

The weights of the PANN could be found with a recursive Least Squares algorithm during training. It is worth noting that in 3. the PANN is shown to lead to better and faster results compared to a normal ANN. The architecture of the PANN model is shown in fig. 1 


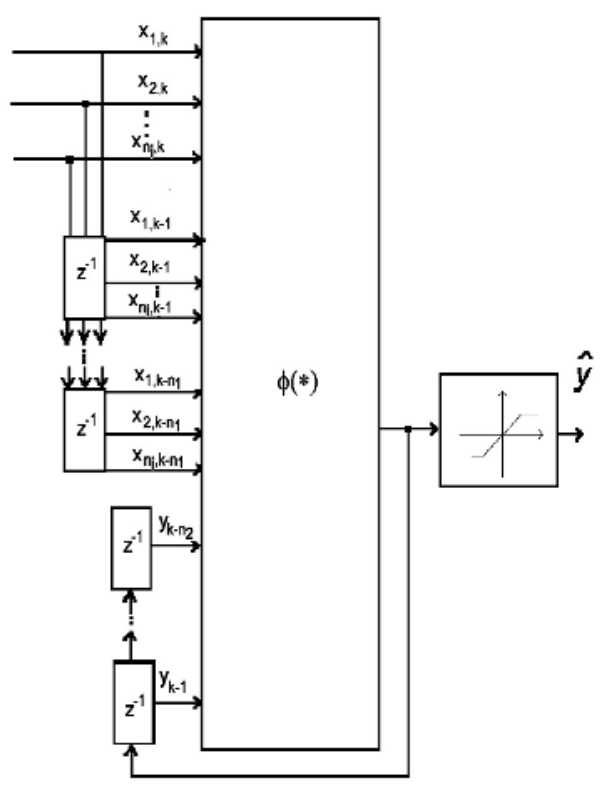

Fig. 1. PANN architecture

\section{Simulations of Theoretical Models}

The output acceleration, velocity and displacements of a one-story shear building (SDOF) are only lateral motion of the mass. In the simulations, two theoretical SDOF were introduced: (a) structure subjected to the Loma Prieta earthquake with mass $m=1 \mathrm{~kg}$, damping $c=1.2566 \mathrm{kgf} \cdot \mathrm{s} / \mathrm{cm}$, and stiffness $k=157.9137$ $\mathrm{kgf} / \mathrm{cm}$; and (b) structure subjected to the Mexico City earthquake with mass $m=1 \mathrm{~kg}$, damping $c=0.3142 \mathrm{kgf} \cdot \mathrm{s} / \mathrm{cm}$, and stiffness $k=9.8696 \mathrm{kgf} / \mathrm{cm}$. In both cases the theoretical acceleration output, sampled at $0.02 \mathrm{sec}$. , was contaminated with $2 \%$ random noise, and the structure was subjected to smooth and compact hysteresis for stability purposes. In this sense, for SDOF (b) the seismic record had to be scaled to $30 \%$ amplitude.

A PANN with $p=2, n_{i}=n_{1}=0$, and $n_{2}=4$ is used for training. Training neural networks is usually based on two criteria: (1) minimizing the error, or (2) by reaching a fixed number of iterations (epochs). Real-time techniques need a different approach due to the fact that the learning process has to be done on-line; thus, training criteria was done with the weight variance herein. One conclusion drawn from the results is that at least two cycles of motion are needed for training because the weight variance tends to zero after that time.

In our simulations, 100 samples (2 seconds) are required for training SDOF (a), and 200 (4 seconds) for SDOF (b). Fig. 2 shows the training and prediction of the hysteretic SDOF (a) in a three-second window. 


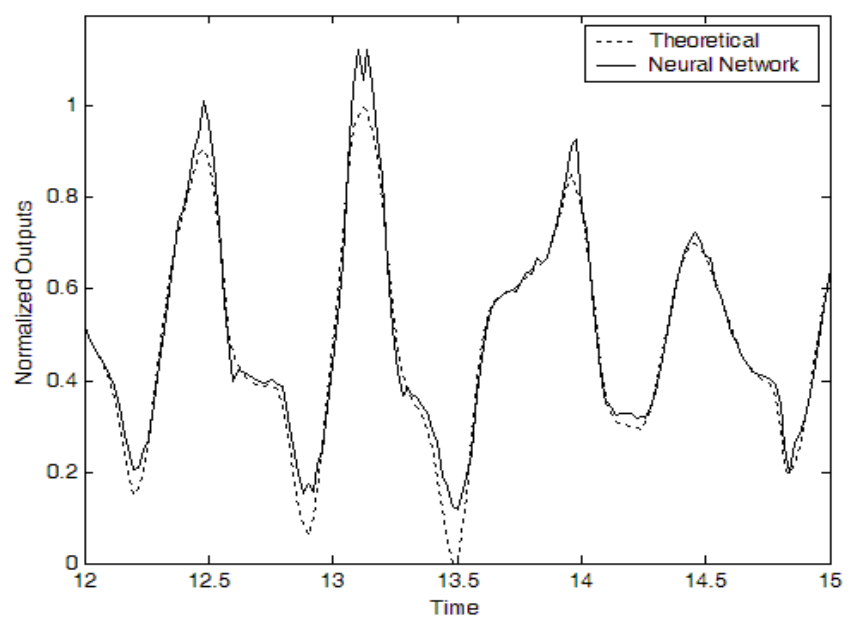

Fig. 2. Prediction of the intense part, Loma Prieta input

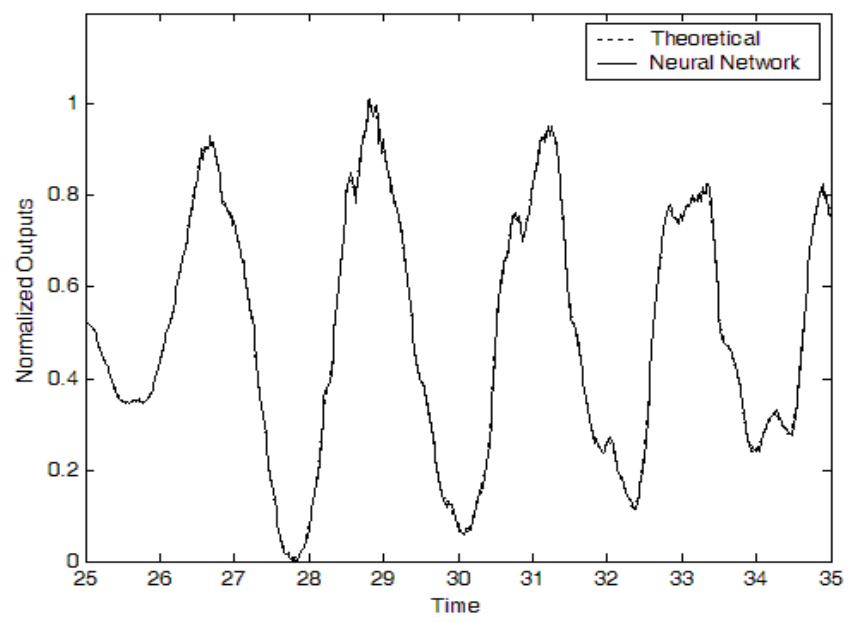

Fig. 3. Prediction of the intense part, Mexico City input

Training could identify a nonlinear model with very small hysteresis, and when the hysteresis cycles become wider at the intense part of the excitation, around 12 seconds of motion, the prediction looses some accuracy. Nonetheless, the proposed PANN is able to predict fairly well the acceleration output. Increasing training time could increase accuracy because hysteretic cycles become wider.

On the other hand, fig. 3 shows the prediction of the hysteretic SDOF (b) in a ten-second window. It is worth noting that the PANN is able to identify very well the nonlinear model, since a bit wider hysteresis occurs from the beginning, and when the intense part takes place the prediction is still very good. 


\section{Identification Using Real Data}

In this section, the PANN is used to identify a model of a real instrumented building. This structure is a 24-story concrete building located in Mexico City. It is instrumented with accelerometer sensors located throughout the building, and several earthquakes have been acquired since its activation. The building has a period of around 3 seconds, thus, training was done with 6 seconds of the output acceleration motion at the centroid of the roof. The seismic event of April 18,2002 , was used for training and prediction.

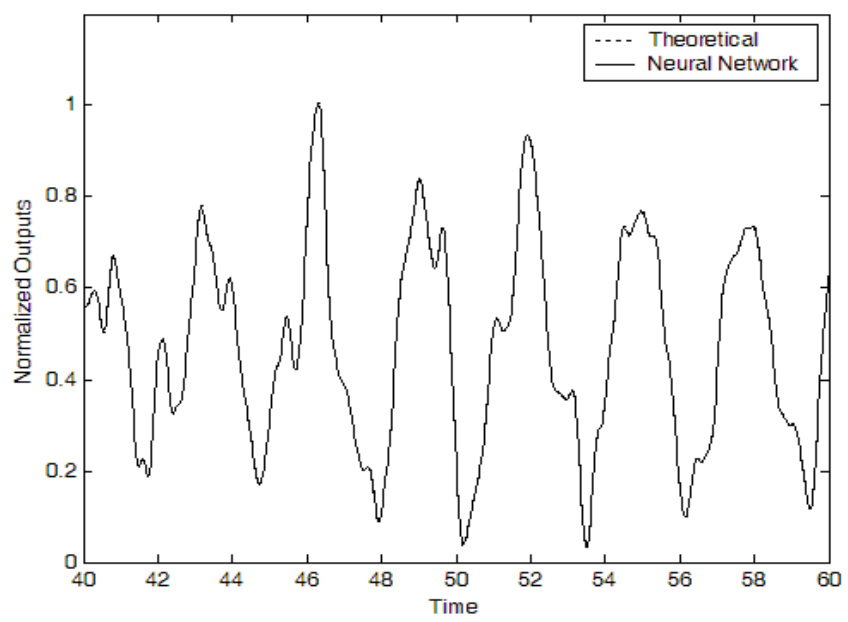

Fig. 4. Prediction of the intense part, April 182002 record

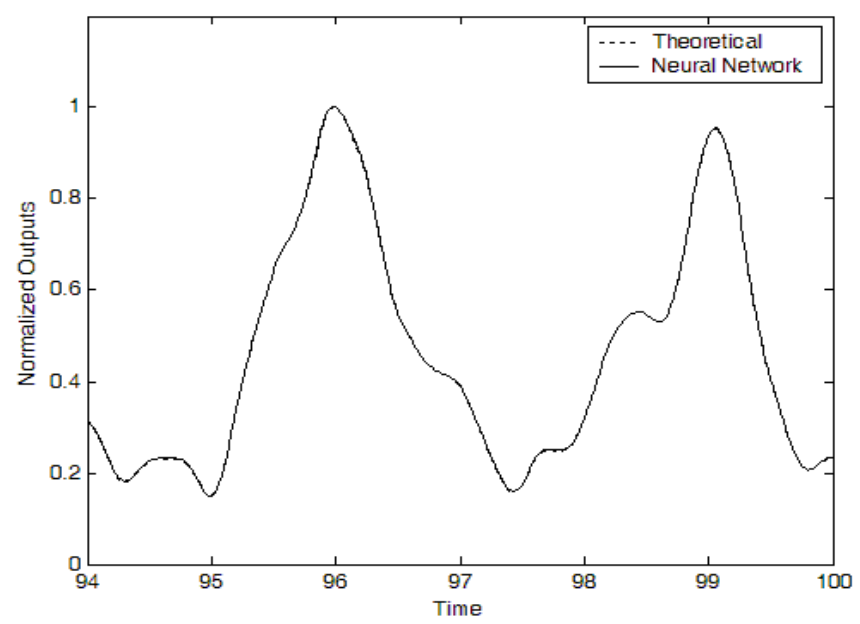

Fig. 5. Prediction of the intese part, January 212003 record 
Fig. 4 shows the prediction of the motion of the building. It is worth noting that both lines seem overlapped due to the fact that the PANN is a very fine tool to identify this structure. The proposed approach is so efficient that no distinction between both lines could be observed.

After training, the weights of the network are kept unchanged to predict the acceleration output for the seismic event of January 21, 2003. Fig. 5 shows the prediction of the motion. Note again that both lines seem overlapped beause the prediction error is very small. In this case, this coud mean that the building has not suffered a noticeable change on its structural properties, since the model still predicts accurately the motion, even after ten months between both seismic events. Therefore, this technique could be used later as a tool for fault detection analysis.

\section{Conclusions}

In the last two decades, several buildings have been instrumented in order to monitor their structural health through the analysis of measured acceleration, velocity and displacement records. The present research proposes the use of a polynomial artificial neural network (PANN) to identify the nonlinear behavior of a building structure, and to forecast the acceleration output. The PANN is trained on-line with only the first two cycles of motion.

To test the effectiveness of the proposed algorithm, two theoretical simulations were introduced. The hysteretic structures were subjected to the seismic records of Loma Prieta (USA, 1989) and Mexico City (Mexico, 1985). The results show fast convergence speed of the weights, and good accuracy to forecast the nonlinear output.

Later on, a model of a real instrumented building was identified with the PANN. The real acquired seismic event of April 18, 2002, was used to train and forecast the motion of the roof.

Finally, the real acquired seismic event of January 21, 2003, was used to predict the motion of the roof using the model identified earlier. Very encouraging results are derived from the analysis. In the long run, the present research is aimed to develop a technique that could be used in conjunction with fault detection analysis, structural health monitoring, and structural control.

\section{References}

1. Box, G. E. P., \& Jenkin, G. M., Time Series Analysis: Forecasting and Control, San Francisco, CA, Holden-Day (1970)

2. Chen, S., \& Billings, A., "Representations of Nonlinear Systems: the NARMAX model", Int. J. of Control, Vol. 49, No. 3 (1989)

3. Gomez-Ramirez, E., Poznyak, A., Gonzalez-Yunes, A., \& Avila-Alvarez, M., "Adaptive Architecture of Polynomial Artificial Neural Network to Forecast Nonlinear Time Series", Congress on Evolutionary Computation, CEC '99, Mayflower, Washington, D.C., USA, July 6 - 9 (1999) 
4. Housner, G. W., Bergman, L. A., Caughey, T. K., Chassiakos, A. G., Claus, R. O., Masri, S. F., Skelton, R. E., Soong, T. T., Spencer, B. F., \& Yao, J. T. P., "Structural Control: Past, Present and Future", Journal of Engineering Mechanics, Vol. 123, No. 9, Sep. (1997)

5. Humar, J. L., Dynamics of Structures, A. A. Balkema Publishers, 2nd Edition (2001)

6. Korenberg, M., Billings, S. A., Liu, Y. P., \& McIlroy, P. J., "Orthogonal Parameter Estimation Algorithm for Non-Linear Stochastic Systems", International Journal of Control, Vol. 48, No. 1 (1988)

7. Kosmatopoulos, E. B., Smyth, A. W., Masri, S. F., \& Chassiakos, A. G., "Robust Adaptive Neural Estimation of Restoring Forces in Nonlinear Structures", Transactions of the ASME, Journal of Applied Mechanics, Vol. 68, November (2001)

8. Loh, C. H., \& Chung, S. T., "A Three-Stage Identification Approach for Hysteretic Systems", Earthquake Engineering and Structural Dynamics, Vol. 22, (1993) 129150

9. Martinez-Garcia, J. C., Gomez-Gonzalez, B., Martinez-Guerra, R., \& RiveroAngeles, F. J., "Parameter Identification of Civil Structures Using Partial Seismic Instrumentation", in 5th Asian Control Conference, ASCC, Melbourne, Australia, July 20-23 (2004)

10. Masri, S. F., Miller, R. K., Saud, A. F., \& Caughey, T. K., "Identification of Nonlinear Vibrating Structures: Part I - Formulation", Transactions of the ASME, J. of Applied Mechanics, Vol. 57, Dec. (1987)

11. Masri, S. F., Chassiakos, A. G., \& Caughey, T. K., "Structure-unknown non-linear dynamic systems: identification through neural networks", Smart Mater. Struct., 1, (1992) 45-56

12. Masri, S. F., Chassiakos, A. G., \& Caughey, T. K., "Identification of nonlinear dynamic systems using neural networks", J. of Applied Mechanics, 60, (1993) 12333

13. Mohammad, K. S., Worden, K., \& Tomlinson, G. R., "Direct Parameter Estimation for Linear and Non-linear Structures", Journal of Sound and Vibration, 152 (3) (1992)

14. Sugeno, M., Industrial Applications of Fuzzy Control, Elsevier Science Pub. Co. (1985)

15. Wen, Y. K., "Method for Random Vibration of Hysteretic Systems", Journal of Engineering Mechanics, ASCE, 102(2), (1976) 249-263

16. Yar, M., \& Hammond, J. K., "Parameter Estimation for Hysteretic Systems", J. of Sound and Vibration, 117 (1) (1987) 